\title{
Stability, Chaos Detection, and Quenching Chaos in the Swing Equation System
}

\author{
Shun-Chang Chang (i) \\ Department of Mechanical and Automation Engineering, Da-Yeh University, No. 168 University Road, Dacun, \\ Changhua 51591, Taiwan \\ Correspondence should be addressed to Shun-Chang Chang; changsc@mail.dyu.edu.tw
}

Received 31 October 2020; Revised 21 November 2020; Accepted 5 December 2020; Published 23 December 2020

Academic Editor: Viet-Thanh Pham

Copyright (C) 2020 Shun-Chang Chang. This is an open access article distributed under the Creative Commons Attribution License, which permits unrestricted use, distribution, and reproduction in any medium, provided the original work is properly cited.

\begin{abstract}
The main objective of this study is to explore the complex nonlinear dynamics and chaos control in power systems. The rich dynamics of power systems were observed over a range of parameter values in the bifurcation diagram. Also, a variety of periodic solutions and nonlinear phenomena could be expressed using various numerical skills, such as time responses, phase portraits, Poincaré maps, and frequency spectra. They have also shown that power systems can undergo a cascade of period-doubling bifurcations prior to the onset of chaos. In this study, the Lyapunov exponent and Lyapunov dimension were employed to identify the onset of chaotic motion. Also, state feedback control and dither signal control were applied to quench the chaotic behavior of power systems. Some simulation results were shown to demonstrate the effectiveness of these proposed control approaches.
\end{abstract}

\section{Introduction}

The characteristics of power systems are well known to be inherently nonlinear owing to the nonlinearity of synchronous generators. The most important issue in power system operation is the prevention of voltage collapse. Various works have already studied the voltage collapse in electric power systems [1-4]. A power system is typically described by a nonlinear dynamical system of equations, including system parameters. Altering one of these parameters changes the power system dynamics that exhibit chaos motion, leading to voltage collapse. However, the chaotic motion in a power system may destabilize it, leading to voltage collapses and even catastrophic blackouts. Modern nonlinear theories of bifurcation and chaos are widely adopted in the studies of nonlinear systems, and chaos dynamics in power systems have been widely studied [5-14]. This work presents several numerical schemes, including bifurcation diagrams, phase portraits, Poincaré maps, and frequency spectra, to clearly explain the rich nonlinear dynamics in power systems. Additionally, the Lyapunov exponents of smooth dynamical systems were calculated using highly developed algorithms [15-17] with the objective of determining whether a system exhibits chaos or not.

Chaotic behaviors in power systems are considered undesirable due to the restrictions they impose on the operating ranges of electrical and mechanical devices. The dynamics of a power system become unstable when they exhibit chaotic motions. If instability is not properly controlled, it causes voltage collapse, eventually leading to blackouts [12]. Hence, in many engineering applications, control approaches are developed to convert chaotic motions into periodic orbits or steady states. Since the pioneering work of Ott et al. [18] in controlling chaos, many modified methods and other approaches have been successively proposed [19-26]. Various control algorithms have also been presented to control the chaos of power systems $[8,27-30]$. This work proposed converting chaotic behaviors into periodic motions to improve the performance of system dynamics with multiple machine power system chaotic behaviors. Also, chaotic motions in a power system were inhibited using state feedback control $[21,31]$ and dither signal control [32], and the simulation results were 
presented to confirm the feasibility and efficacy of the proposed control approaches.

\section{The Problem Description and Modeling of Special Swing Equations for Three Machines}

A power system model of three synchronous generators with a resistive load configuration was considered, as shown in Figure 1. Synchronous generators are the most important energy source for power systems. The study of interconnected power systems with synchronous motors as significant equipment is the key to studying the dynamic characteristics of power systems. The governing differential equations of the power system in Figure 1 can be expressed as follows $[33,34]$ :

$$
\begin{aligned}
\dot{\delta}_{1} & =\dot{\omega}_{1}, \\
M_{1} \dot{\omega}_{1} & =-D_{1} \omega_{1}+P_{1}-F_{12} \sin \left(\delta_{1}-\delta_{2}\right)-F_{13} \sin \left(\delta_{1}-\delta_{3}\right), \\
\dot{\delta}_{2} & =\dot{\omega}_{2}, \\
M_{2} \dot{\omega}_{2} & =-D_{2} \omega_{2}+P_{2}-F_{21} \sin \left(\delta_{2}-\delta_{1}\right)-F_{23} \sin \left(\delta_{2}-\delta_{3}\right), \\
\dot{\delta}_{3} & =\dot{\omega}_{3}, \\
M_{3} \dot{\omega}_{3} & =-D_{3} \omega_{3}+P_{3}-F_{31} \sin \left(\delta_{3}-\delta_{1}\right)-F_{32} \sin \left(\delta_{3}-\delta_{2}\right) .
\end{aligned}
$$

A special case of the swing equations was considered for three machines. It was assumed that machine 1 has a large inertia, i.e., $M_{1}=\left(\bar{M}_{1} / \varepsilon\right)$ and $\varepsilon \ll 1$. The transmission line joining machines 2 and 3 is shorter than that of the other lines. Similarly, the external power $P_{1}$ is proportionally larger: $P_{1}=\left(\bar{P}_{1} / \varepsilon\right)$. With these assumptions, the conservative swing equations for the three machines are

$$
\dot{\delta}_{1}=\dot{\omega}_{1}
$$

$\bar{M}_{1} \dot{\omega}_{1}=-\varepsilon D_{1} \omega_{1}+\bar{P}_{1}-\varepsilon F_{12} \sin \left(\delta_{1}-\delta_{2}\right)-\varepsilon F_{13} \sin \left(\delta_{1}-\delta_{3}\right)$,

$$
\dot{\delta}_{2}=\dot{\omega}_{2}
$$

$$
M_{2} \dot{\omega}_{2}=-D_{2} \omega_{2}+P_{2}-F_{21} \sin \left(\delta_{2}-\delta_{1}\right)-F_{23} \sin \left(\delta_{2}-\delta_{3}\right) \text {, }
$$

$$
\dot{\delta}_{3}=\dot{\omega}_{3}
$$

$M_{3} \dot{\omega}_{3}=-D_{3} \omega_{3}+P_{3}-F_{31} \sin \left(\delta_{3}-\delta_{1}\right)-F_{32}\left(\delta_{3}-\delta_{2}\right)$.

From $[33,34], \delta_{1}$ can be expressed as follows:

$$
\delta_{1}=-\varepsilon \mu_{2} \delta_{2}-\varepsilon \mu_{3} \delta_{3},
$$

where $\mu_{2}=\left(M_{2} / \bar{M}_{1}\right)$ and $\mu_{3}=\left(M_{3} / \bar{M}_{1}\right)$.

By substituting equation ( 3 ) in equations (2a)-(2f), write an autonomous differential equation for $\delta_{2}, \delta_{3}, \omega_{2}$, and $\omega_{3}$ and eliminate $\delta_{1}$ and $\omega_{1}$ as follows:

$$
\begin{aligned}
\dot{\delta}_{2}= & \dot{\omega}_{2}, \\
\dot{\omega}_{2}= & -D_{2} \omega_{2}+\bar{\alpha}_{2}-\beta_{21} \sin \left[\delta_{2}\left(1+\varepsilon \mu_{2}\right)+\varepsilon \mu_{3} \delta_{3}\right] \\
& -\beta_{23} \sin \left(\delta_{2}-\delta_{3}\right), \\
\dot{\delta}_{3}= & \dot{\omega}_{3}, \\
\dot{\omega}_{3}= & -D_{3} \omega_{3}+\bar{\alpha}_{3}-\beta_{31} \sin \left[\delta_{2}\left(1+\varepsilon \mu_{2}\right)+\varepsilon \mu_{3} \delta_{3}\right] \\
& -\beta_{32} \sin \left(\delta_{3}-\delta_{2}\right),
\end{aligned}
$$

where $\bar{\alpha}_{2}=\left(P_{2} / M_{2}\right), \quad \beta_{21}=\left(F_{21} / M_{2}\right), \quad \beta_{23}=\left(F_{23} / M_{2}\right)$, $\bar{\alpha}_{3}=\left(P_{3} / M_{3}\right), \beta_{31}=\left(F_{31} / M_{3}\right)$, and $\beta_{32}=\left(F_{32} / M_{3}\right)$.

For convenience, let $\varepsilon=0$, and simplify equations (4a)-(4d) into

$$
\begin{aligned}
& \dot{\delta}_{2}=\dot{\omega}_{2}, \\
& \dot{\omega}_{2}=-D_{2} \omega_{2}+\bar{\alpha}_{2}-\beta_{21} \sin \left(\delta_{2}\right)-\beta_{23} \sin \left(\delta_{2}-\delta_{3}\right), \\
& \dot{\delta}_{3}=\dot{\omega}_{3}, \\
& \dot{\omega}_{3}=-D_{3} \omega_{3}+\bar{\alpha}_{3}-\beta_{31} \sin \left(\delta_{3}\right)-\beta_{32} \sin \left(\delta_{3}-\delta_{2}\right) .
\end{aligned}
$$

From $[33,34]$, express $\bar{\alpha}_{k}$ as follows:

$$
\bar{\alpha}_{k}=\bar{P}_{k}-K_{f} \omega_{k}, \quad k=2,3,
$$

where $P_{k}$ is a constant real power and $K_{f}=L_{k} / M_{k}$. $L_{k}$ is a load-frequency coefficient. By substituting equation (6) in (5a)-(5d),

$$
\dot{\delta}_{2}=\dot{\omega}_{2}
$$

$$
\dot{\omega}_{2}=-D_{2} \omega_{2}+\bar{P}_{2}-K_{f} \omega_{2}-\beta_{21} \sin \left(\delta_{2}\right)-\beta_{23} \sin \left(\delta_{2}-\delta_{3}\right),
$$

$$
\dot{\delta}_{3}=\dot{\omega}_{3}
$$

$$
\dot{\omega}_{3}=-D_{3} \omega_{3}+\bar{P}_{3}-K_{f} \omega_{3}-\beta_{31} \sin \left(\delta_{3}\right)-\beta_{32} \sin \left(\delta_{3}-\delta_{2}\right) \text {. }
$$

With $y_{1}=\delta_{2}, y_{2}=\omega_{2}, y_{3}=\delta_{3}$, and $y_{4}=\omega_{3}$ as the state variables, the state equations of the swing equation can be written as follows: 


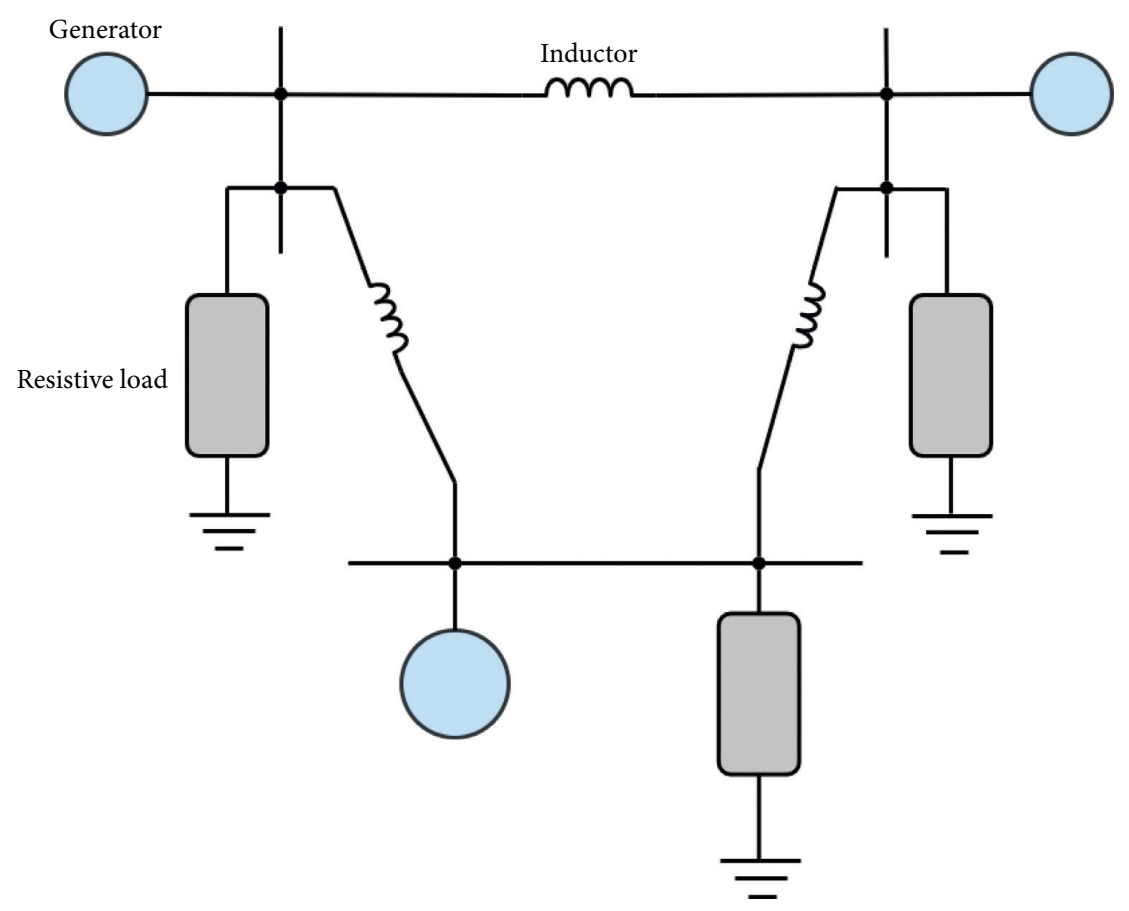

FIGURE 1: Schematic of a power system.

$$
\dot{y}_{1}=y_{2}
$$

$$
\dot{y}_{2}=\bar{P}_{2}-\left(D_{2}+K_{f}\right) y_{2}-\beta_{21} \sin \left(y_{1}\right)-\beta_{23} \sin \left(y_{1}-y_{3}\right),
$$

$$
\begin{aligned}
& \dot{y}_{3}=y_{4}, \\
& \dot{y}_{4}=\bar{P}_{3}-\left(D_{3}+K_{f}\right) y_{4}-\beta_{31} \sin \left(y_{3}\right)-\beta_{32} \sin \left(y_{3}-y_{1}\right) .
\end{aligned}
$$

Table 1 [31] lists the numerical values for all the parameters in equations $(8 \mathrm{a})-(8 \mathrm{~d})$.

\section{Overall Characteristics of the Power System: Simulation Results and Discussion}

Numerical simulations were executed based on equations (8a)-(8d) to clearly understand the overall characteristics of the power system. The commercial package DIVPRK of IMSL in the FORTRAN subroutines was utilized for the mathematic applications to solve the ordinary differential equation problems [35]. Figure 2 presents the resulting bifurcation diagram, which clearly reveals that the first period-doubling bifurcation occurred at about $K_{f}=0.078$ and that a chaotic motion appeared approximately below $K_{f}=0.0109$. Figures 3-6 display various responses exhibited by this system in detail, where each type of response was characterized in detail using a phase portrait, a Poincaré map, and a frequency spectrum. The equilibrium point of equations ( $8 \mathrm{a})-(8 \mathrm{~d})$ was stable with $K_{f}>0.078$, revealing that no chatter vibration occurred. Figures $3(a)-3(d)$ show the period-1 motion. Also, Figures 4(a)-4(d) show a cascade of period-doubling bifurcations with new frequency components at $\Omega / 2,3 \Omega / 2,5 \Omega / 2$. . , causing a series of subharmonic components. Figures 5(a)-5(d) depict the first period-four bifurcation, which occurred when $K_{f}$ fell below $K_{f} \approx 0.0275$. A cascade of chaos-inducing period-doubling bifurcations then appeared as $K_{f}$ continued to fall in Figure 2, resulting in a chatter vibration that could cause a voltage collapse, thereby significantly reducing the power system performance and possibly causing catastrophic blackouts. Two descriptors, the Poincaré map and frequency spectrum, could be utilized to characterize the essence of the chaotic behavior. The Poincaré map shows an infinite set of points called a strange attractor. Simultaneously, the frequency spectrum of the chaotic motion is a continuous broad spectrum. These two main features, strange attractors and continuous-type Fourier spectrum, are strong indicators of chaos. Figures 6(a)-6(d) clearly reveal the chaotic behavior in detail.

\section{Lyapunov Exponent and Lyapunov Dimension for Analyzing the Chaos in the Power System}

As described in Section 3, the chaotic motion in power systems is difficult to identify using traditional approaches. This section describes the use of Lyapunov exponents to verify the occurrence of chaos in power systems. Every dynamic system involves a spectrum of Lyapunov exponents $(\lambda)[15]$, indicating changes in the length, area, and volume within a phase space. To determine whether a system exhibits the characteristics of chaos, only the largest Lyapunov exponent needs to be calculated to determine 
TABLe 1: Physical parameters of a power system.

\begin{tabular}{lc}
\hline Parameter & Value \\
\hline$\beta_{21}$ & -2 \\
$\beta_{23}$ & -1 \\
$\beta_{31}$ & -1 \\
$\beta_{32}$ & -1 \\
$D_{2}$ & 0.2 \\
$D_{3}$ & 0.5 \\
$\bar{P}_{2}$ & 0.3 \\
$\bar{P}_{3}$ & 0.5 \\
\hline
\end{tabular}

whether nearby trajectories diverge $(\lambda>0)$ or converge $(\lambda<0)$ on average. Any bounded motion in a system with at least one positive Lyapunov exponent is defined as chaotic, whereas periodic motions exhibit no positive Lyapunov exponents.

Figure 7 plots the evolutions of the largest Lyapunov exponent in the power system, as computed using the algorithm proposed by Wolf et al. [15]. This figure reveals that the onset of the chaotic motion occurred at approximately $K_{f}=0.0109$. At point $\mathbf{P}_{\mathbf{3}}$, the sign of the largest Lyapunov exponent changed from negative to positive as the parameter $K_{f}$ slowly decreased. At points $\mathbf{P}_{\mathbf{1}}$ and $\mathbf{P}_{\mathbf{2}}$, the largest Lyapunov exponents approached zero, which is beyond the point at which the system can undergo bifurcation. Nonetheless, the Lyapunov exponent at that point provided no indication of the involved bifurcation type, thereby necessitating the application of a bifurcation diagram, as shown in Figure 2. A conducted comparison of Figures 7 and 2 indicated the occurrence of period-2 bifurcation at $\mathbf{P}_{\mathbf{1}}$ and period-4 bifurcation at $\mathbf{P}_{\mathbf{2}}$. When $K_{f}=0.09$, the Lyapunov exponents obtained using equations (8a)-(8d) were $\lambda_{1}=-0.000084, \quad \lambda_{2}=-0.0157341, \quad \lambda_{3}=-0.5120906$, and $\lambda_{4}=-0.5977934$, and their sum was $\lambda_{1}+\lambda_{2}+\lambda_{3}+\lambda_{4}=-1.1257021$, which is negative, indicating that the power system had stable periodic motion. By denoting $\lambda_{1} \geq \cdots \geq \lambda_{n}$ as the Lyapunov exponents of a dynamical system, Kaplan and Yorke [36] expressed the estimate of the Lyapunov dimension $d_{L}$ as

$$
d_{L}=j+\frac{1}{\left|\lambda_{j+1}\right|} \sum_{i=1}^{j} \lambda_{i}, \quad \text { where } \sum_{i=1}^{j} \lambda_{i}>0 \text { and } \sum_{i=1}^{j+1} \lambda_{i}<0 \text {. }
$$

Thus, the Lyapunov dimension is an integer for periodic orbits and a noninteger for chaotic motion. Using equations (8a) $-(8 \mathrm{~d})$ with $K_{f}=0.09$, this calculation yielded a Lyapunov dimension of $d_{L}=1$. Therefore, this system exhibited periodic motion, as the Lyapunov dimension was an integer. When the parameter $K_{f}$ continuously decreased across bifurcation point $\mathbf{P}_{\mathbf{3}}$, for example, $K_{f}=0.009$, the Lyapunov exponents were $\lambda_{1}=0.0340716, \quad \lambda_{2}=-0.0000068, \quad \lambda_{3}=-0.3985904$, and $\lambda_{4}=-0.5270599$, and the Lyapunov dimension was $d_{L}=2.0854$. Obviously, the power system could be demonstrated to exhibit chaotic motion, as the largest Lyapunov exponent was positive and the Lyapunov dimension was a noninteger.

\section{Quenching Chaos in the Power System}

Analyzing and predicting the behaviors of chaotic systems are beneficial, but a system needs to be controlled to maximize its benefits. Improving the performance of a dynamic system and avoiding chaotic motion both require periodic motion, which is more important when working under specific conditions. This section presents two control methods, state feedback control $[21,31]$ and dither control [32], to suppress the chaos in the used power system in this study.

5.1. State Feedback Control. Cai et al. [21, 31] proposed a simple and effective method for converting chaos into periodic motion at a steady state using the linear-state feedback of an available system variable. For an $n$-dimensional dynamic system, this method can be briefly summarized as follows:

$$
\dot{x}=f(x, t)
$$

where $x(t) \in R^{n}$ is the state vector and $f=\left(f_{1}, \ldots, f_{i}, \ldots, f_{n}\right)$, where $f_{i}$ is a linear or nonlinear function and $f$ includes at least one nonlinear function. If $f_{k}(x, t)$ is the key nonlinear function that leads to chaotic motion in equation (10), only one term of the state feedback of an available system variable $x_{m}$ was added to the equation that includes $f_{k}(x, t)$ as follows:

$$
\dot{x}_{k}=f_{k}(x, t)+K x_{m}, \quad k, m \in\{1,2, \ldots, n\},
$$

where $K$ is the feedback gain, and the other functions keep their original forms.

Equations (8a)-(8d) can be rewritten as follows with the state feedback control:

$$
\begin{aligned}
& \dot{y}_{1}=y_{2}, \\
& \dot{y}_{2}=\bar{P}_{2}-\left(D_{2}+K_{f}\right) y_{2}-\beta_{21} \sin \left(y_{1}\right)-\beta_{23} \sin \left(y_{1}-y_{3}\right)+K y_{2},
\end{aligned}
$$

$\dot{y}_{3}=y_{4}$

$\dot{y}_{4}=\bar{P}_{3}-\left(D_{3}+K_{f}\right) y_{4}-\beta_{31} \sin \left(y_{3}\right)-\beta_{32} \sin \left(y_{3}-y_{1}\right)+K y_{4}$

Without the state feedback control, equations (8a)-(8d) exhibited chaotic behavior under the parameter $K_{f}=0.009$. Considering that the effect of the state feedback control was 


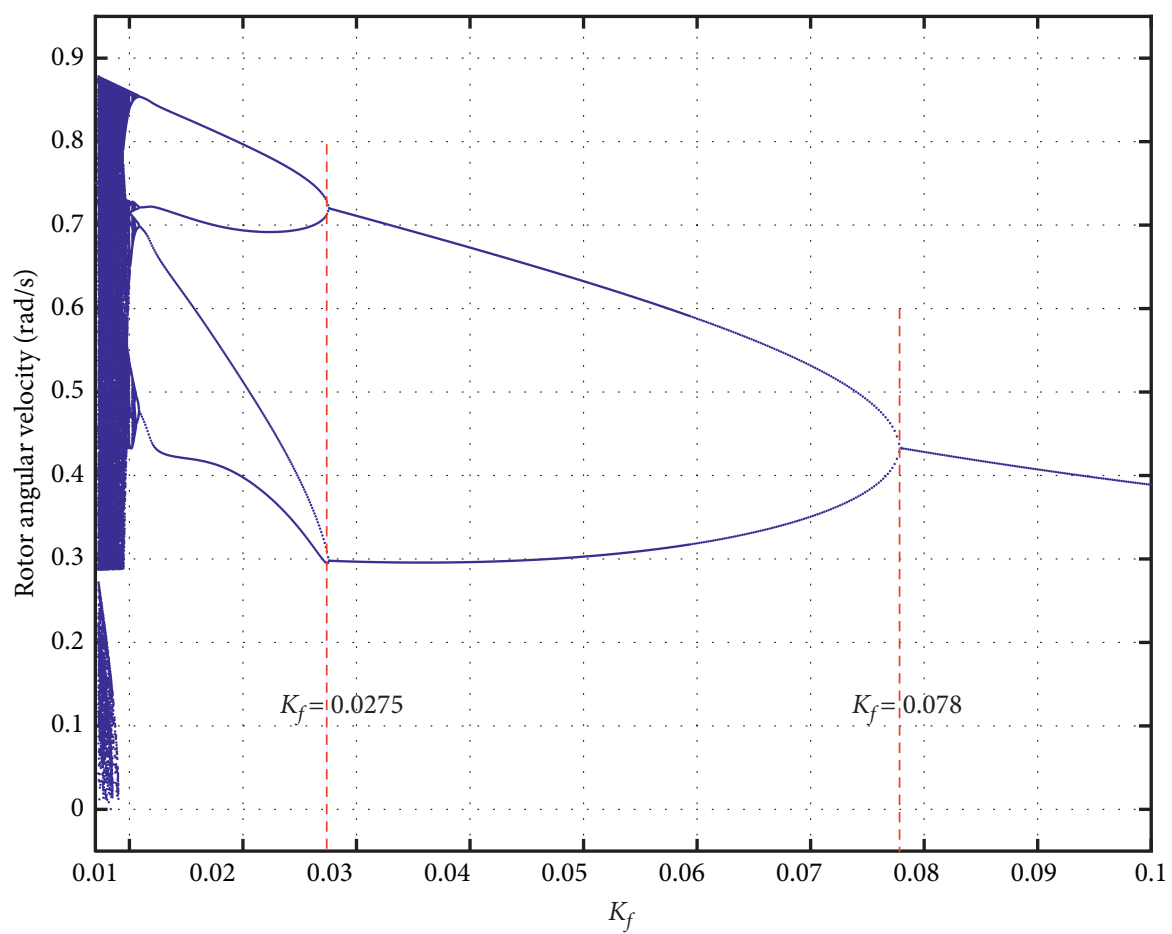

FIGURE 2: Bifurcation diagram of the rotor angular velocity against $K_{f}$.

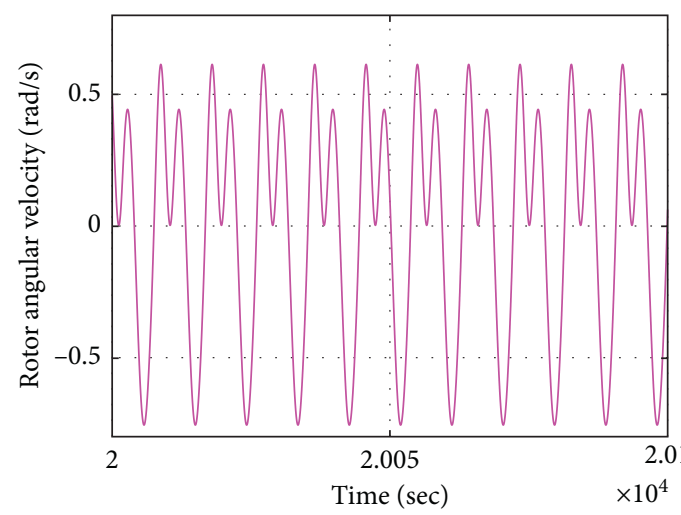

(a)

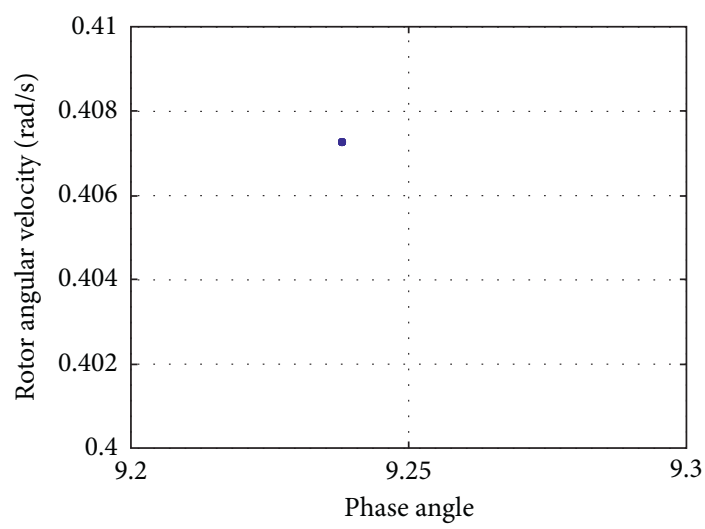

(c)

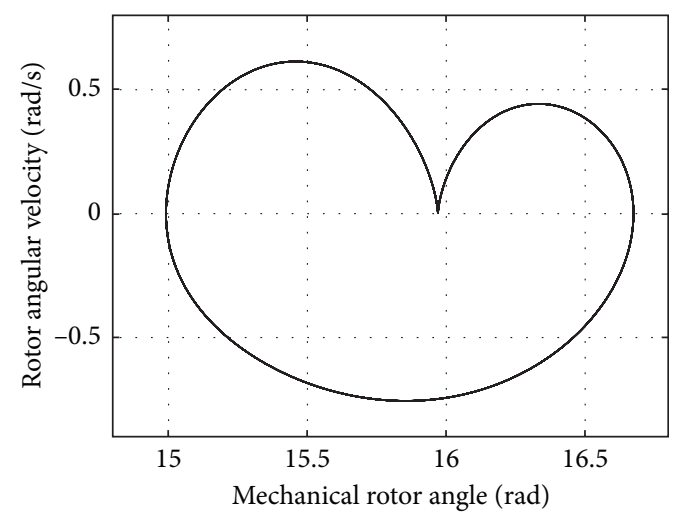

(b)

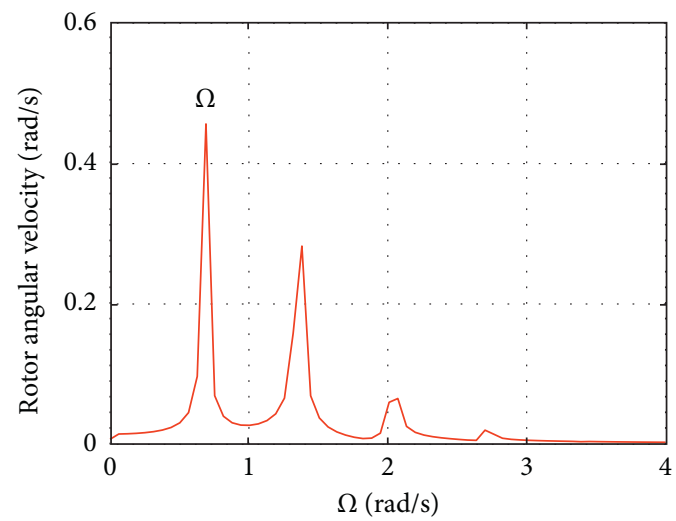

(d)

Figure 3: Period-1 orbit of the numerical simulation results for $K_{f}=0.09$ : (a) time responses; (b) phase portrait; (c) Poincaré map; (d) frequency spectrum. 


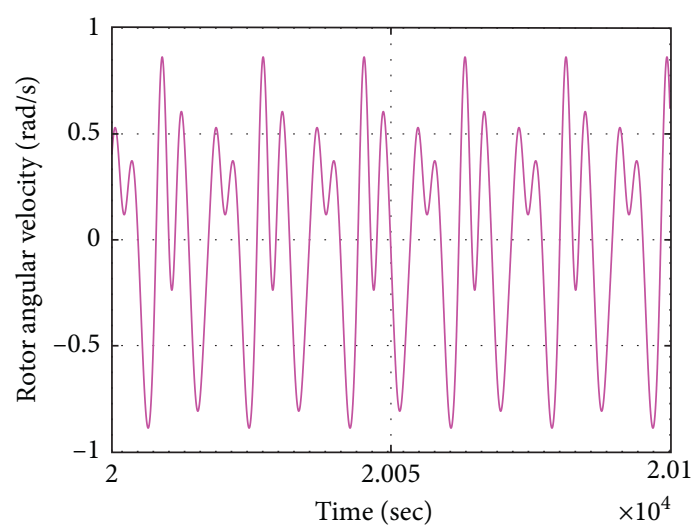

(a)

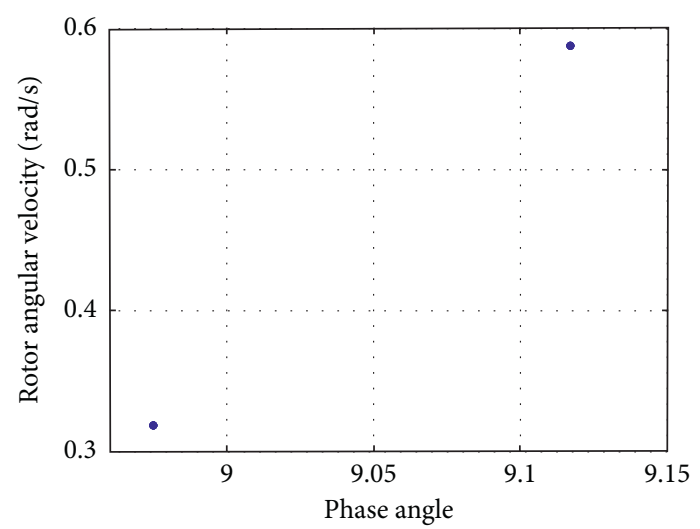

(c)

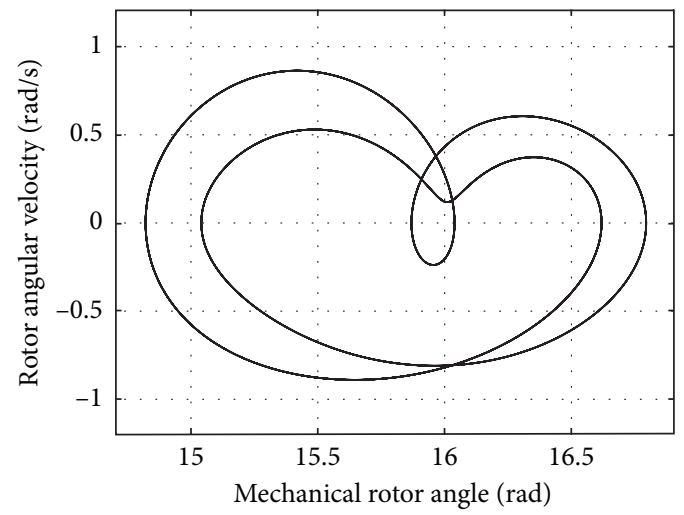

(b)

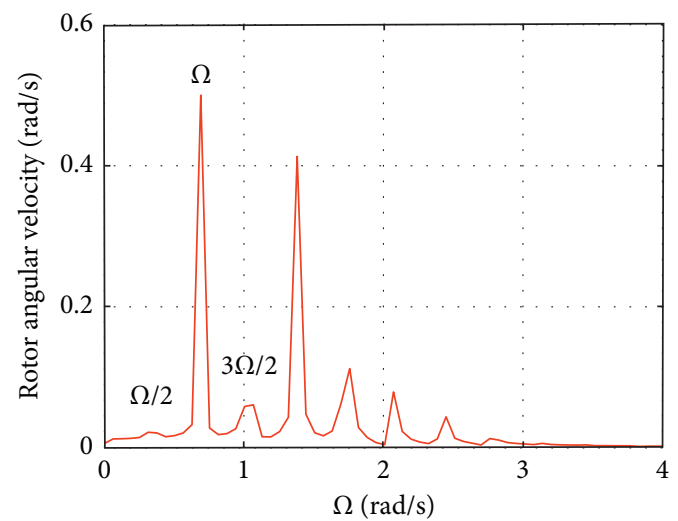

(d)

FIgURe 4: Period-2 orbit of the numerical simulation results for $K_{f}=0.06$ : (a) time responses; (b) phase portrait; (c) Poincaré map; (d) frequency spectrum.

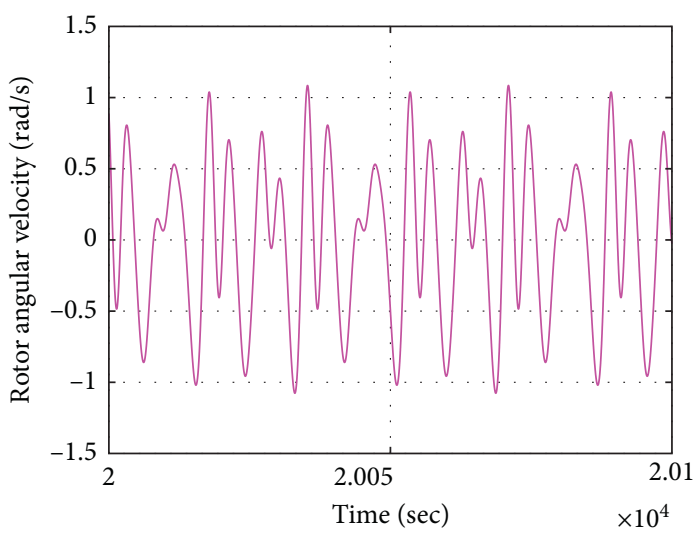

(a)

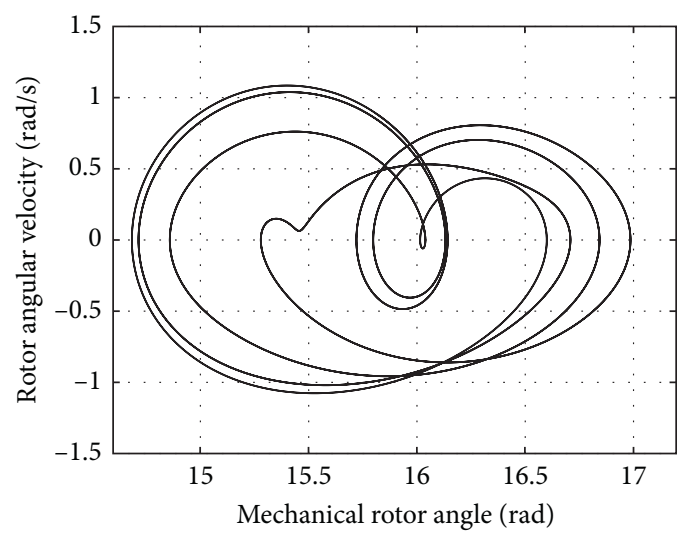

(b)

FIgURe 5: Continued. 


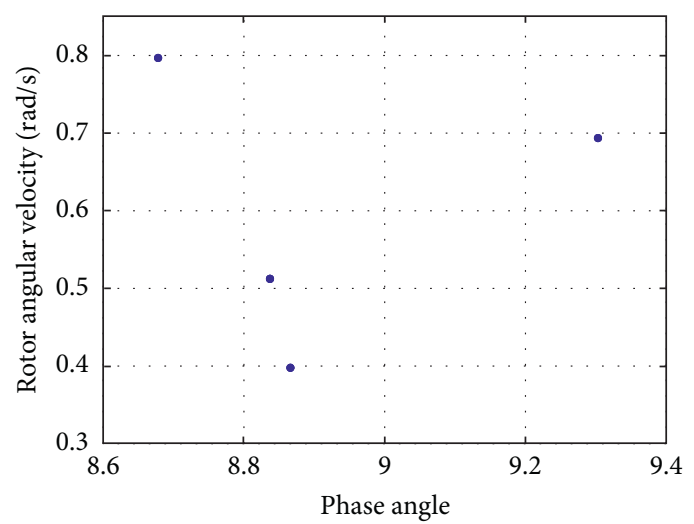

(c)

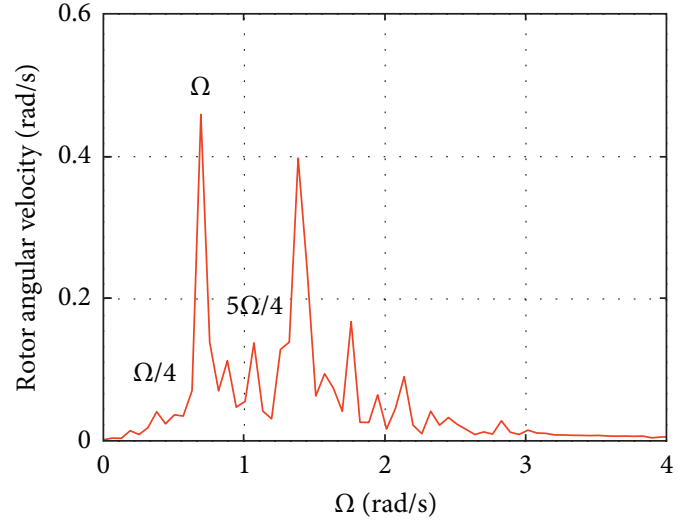

(d)

FIgURE 5: Period-4 orbit of the numerical simulation results $K_{f}=0.02$ : (a) time responses; (b) phase portrait; (c) Poincaré map; (d) frequency spectrum.

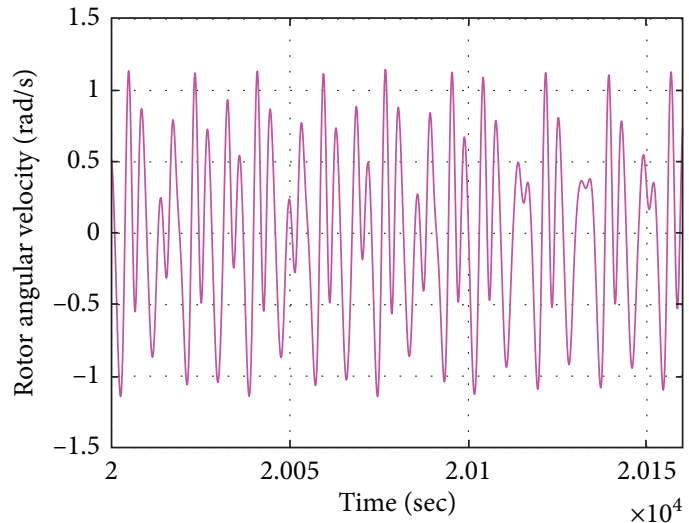

(a)

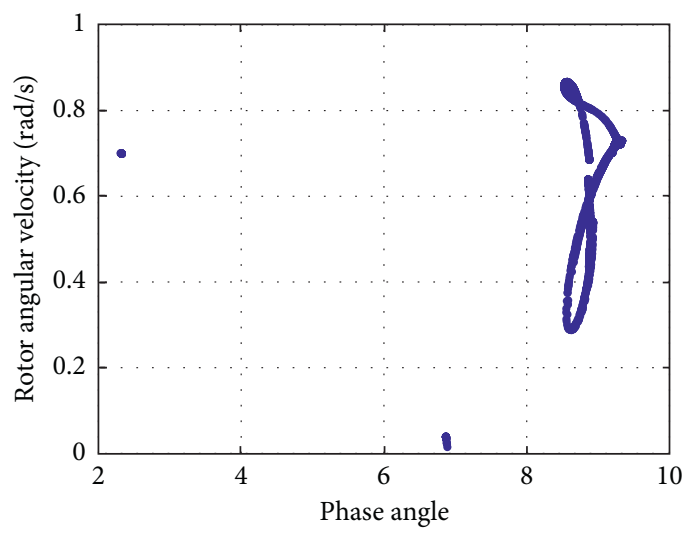

(c)

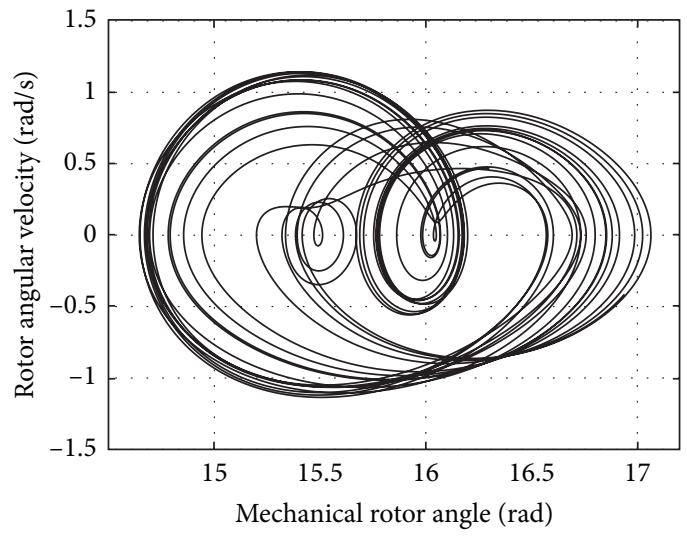

(b)

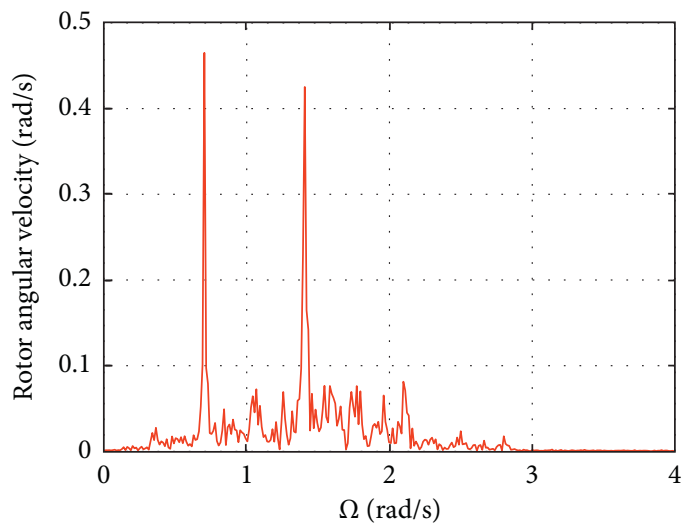

(d)

Figure 6: Chaotic motion of the numerical simulation results for $K_{f}=0.009$ : (a) time responses; (b) phase portrait; (c) Poincaré map; (d) frequency spectrum.

added to the right-hand side of equations (8a)-(8d), by decreasing the feedback gain $K$ from 0 to -0.1 , the chaotic behavior disappeared at certain feedback gains. Figure 8 presents the resulting bifurcation diagram, which comprehensively explains the dynamic behavior of the controlled power system over a range of feedback gains. Chaotic motion appeared when $K<-0.0008$, and stable periodic motion appeared when $K$ decreased beyond -0.0008 . Period-doubling bifurcations 


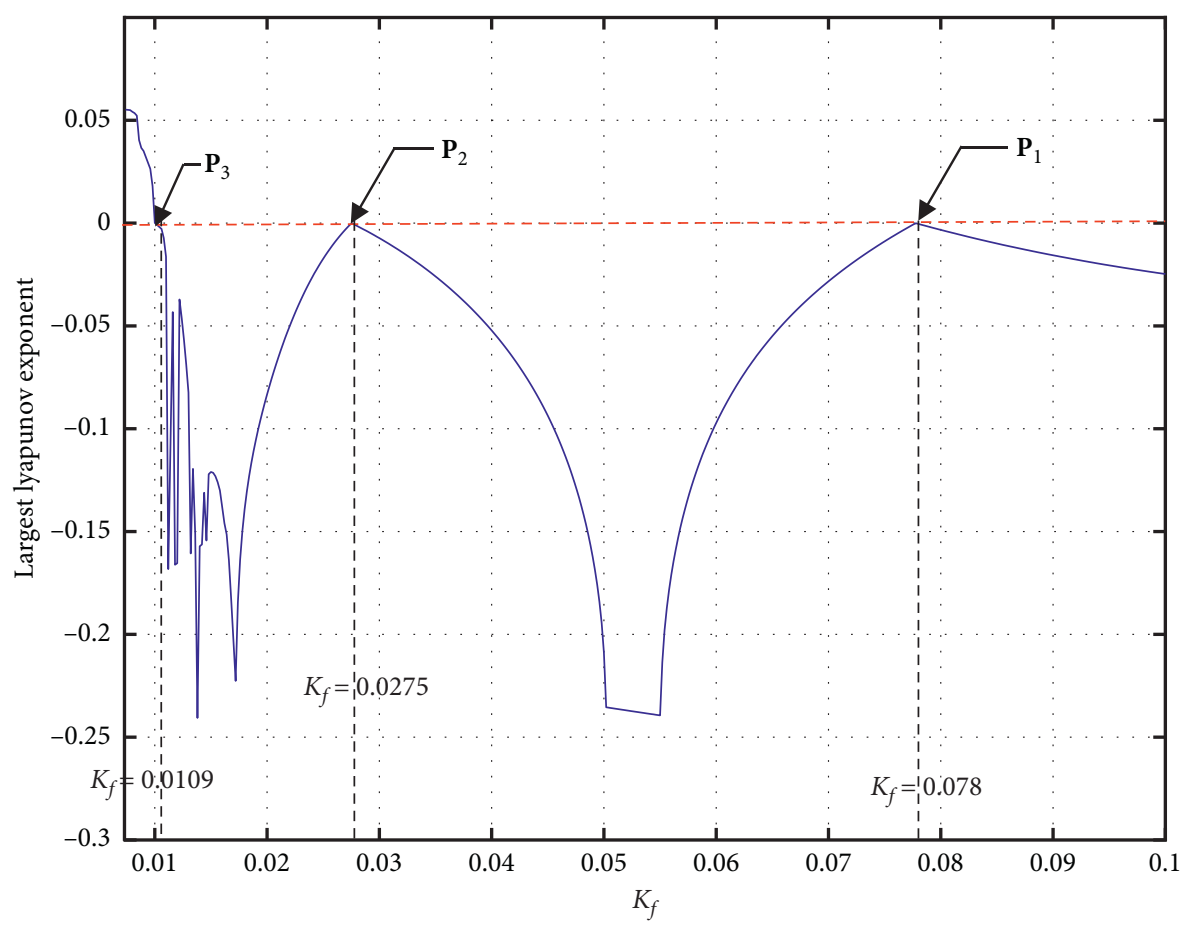

Figure 7: Evolutions of the largest Lyapunov exponent versus $K_{f}$.

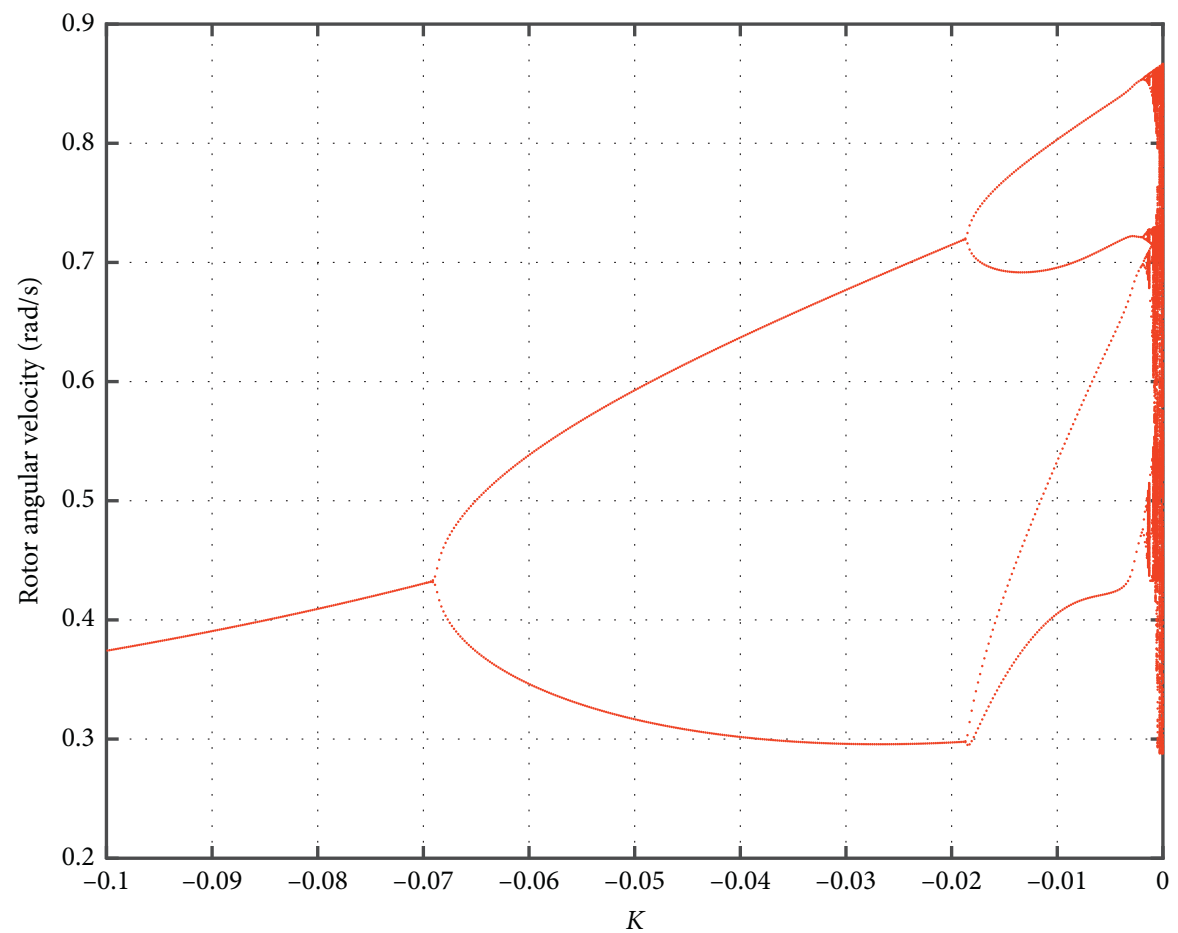

FIGURE 8: Bifurcation diagram of the rotor angular velocity against $K$ for the power system with the state feedback control, where $K$ indicates the feedback gain. 


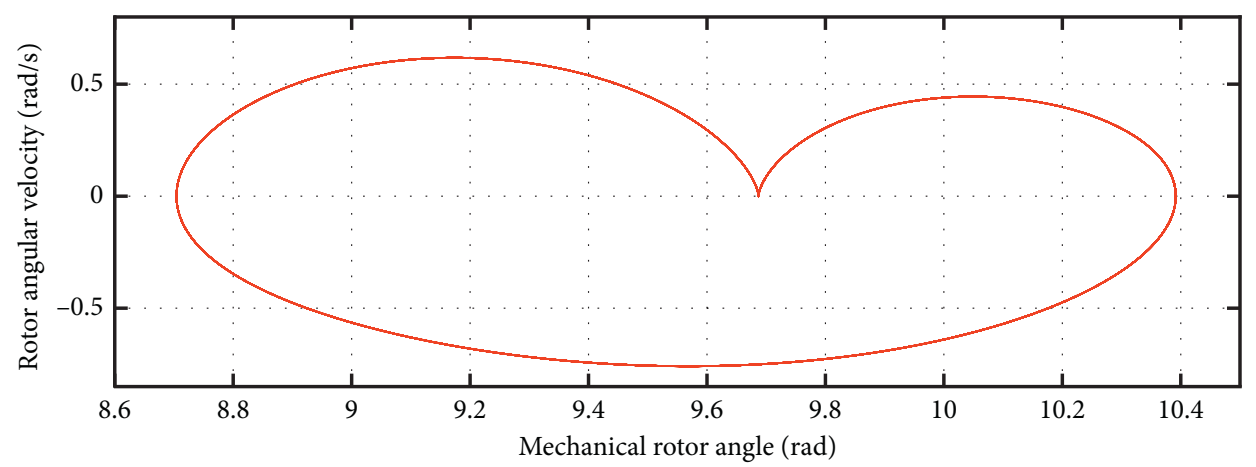

(a)

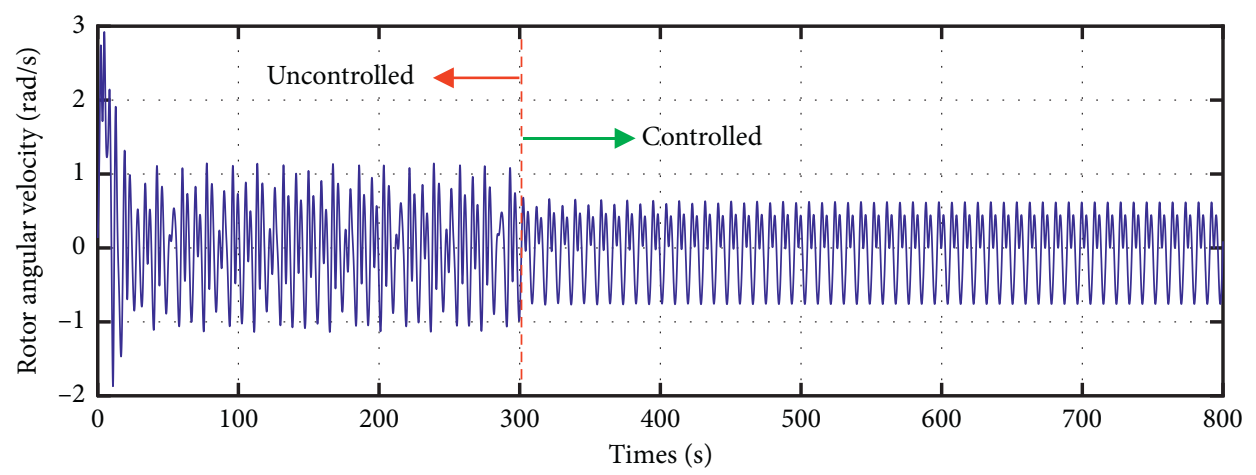

(b)

Figure 9: Transforming chaotic motion into a period- 1 orbit for $K=-0.08$ and $K_{f}=0.009$ : (a) phase portrait of the controlled system; (b) time responses of the controlled system. A state feedback control signal was introduced after $300 \mathrm{~s}$.

appeared when $K$ decreased to between about -0.0699 and -0.0008 . A further decrease in $K$ beyond -0.0699 resulted in a period-1 motion. The efficacy of the proposed system in controlling chaos was demonstrated by applying a control signal after 300 seconds, as shown in Figure 9. Therefore, to suppress the occurrence of chaos, the simple state feedback of an available system variable can be sufficient to disturb the balance of dynamic behaviors in a chaotic system.

5.2. Dither Control. This section describes how to control motion in a chaotic system by injecting another external input dither signal to only modify nonlinear terms. A dither signal averages nonlinearity due to its high frequency and periodic nature. Researchers have developed dither smoothing methods $[32,37]$ to stabilize chaotic systems, and popular dither signals were proposed in [38]. The simplest dither signal is a square-wave dither signal in which the frequency and amplitude are $2000 \mathrm{rad} / \mathrm{s}$ and $W$, respectively, in front of the nonlinearity $f($.$) . Therefore, the effective value$ of $\mu$ and the output of the nonlinear element can be expressed as

$$
\mu=\frac{1}{2}[f(y+W)+f(y-W)] .
$$

Consequently, the system equation can be expressed as

$$
\dot{y}=\mu \text {. }
$$

Considering the influence of the dither signal control added to systems $(8 \mathrm{a})-(8 \mathrm{~d})$ under the parameter $K_{f}=0.009$, by raising the amplitude of the square-wave dither signal from $W=0$ to $W=0.5$, the dynamics changed from chaotic behaviors to periodic motions. Figure 10 displays the evolution of the bifurcation diagram. Figure 11(a) depicts the time response of the displacement with the amplitude of the square-wave dither signal $W=0.2$. The chaotic behavior system was transformed into a period-1 orbit. Figure 11(b) illustrates the phase portrait of the controlled system. Notably, the system exhibited a chaotic behavior before dither was introduced but exhibited a periodic motion afterward. 


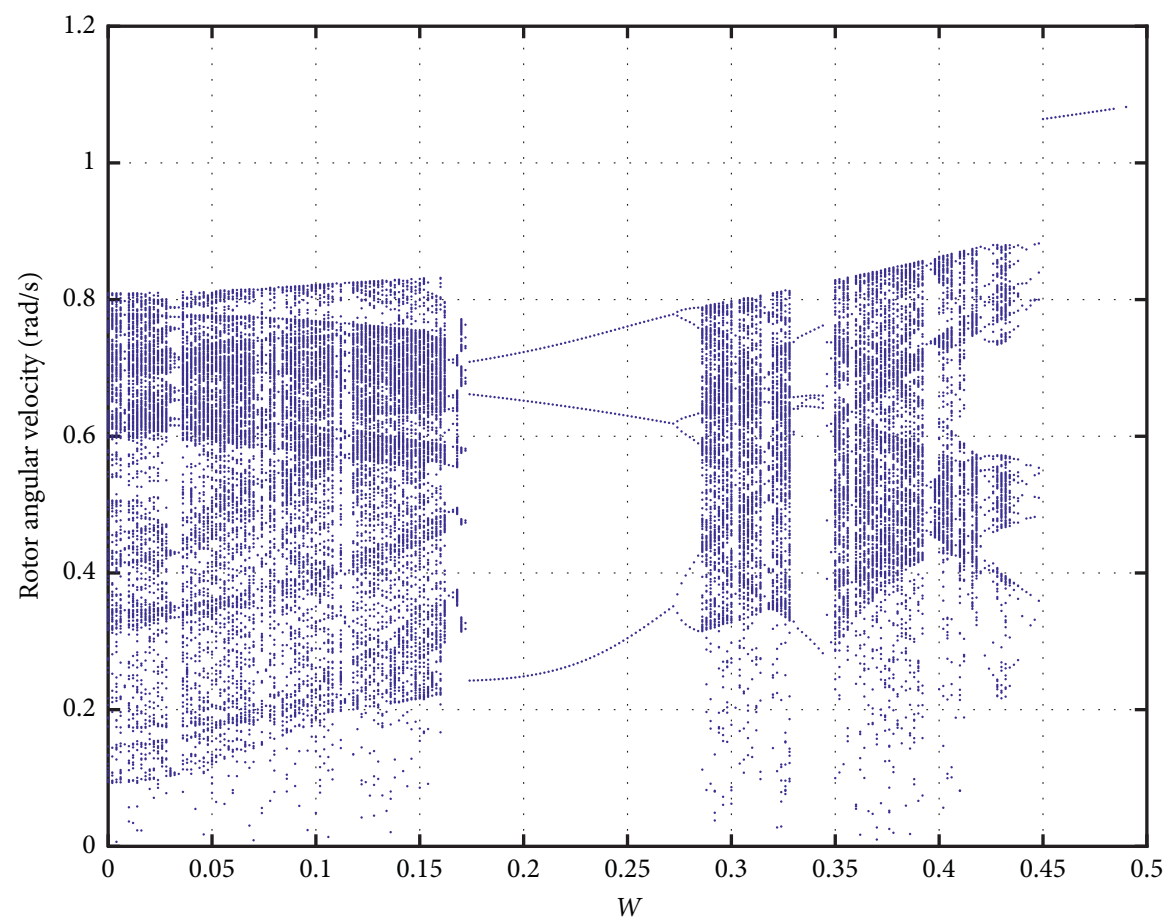

FIGURE 10: Bifurcation diagram of the rotor angular velocity against $W$ for the power system with square-wave dither, where $W$ represents the dither amplitude.

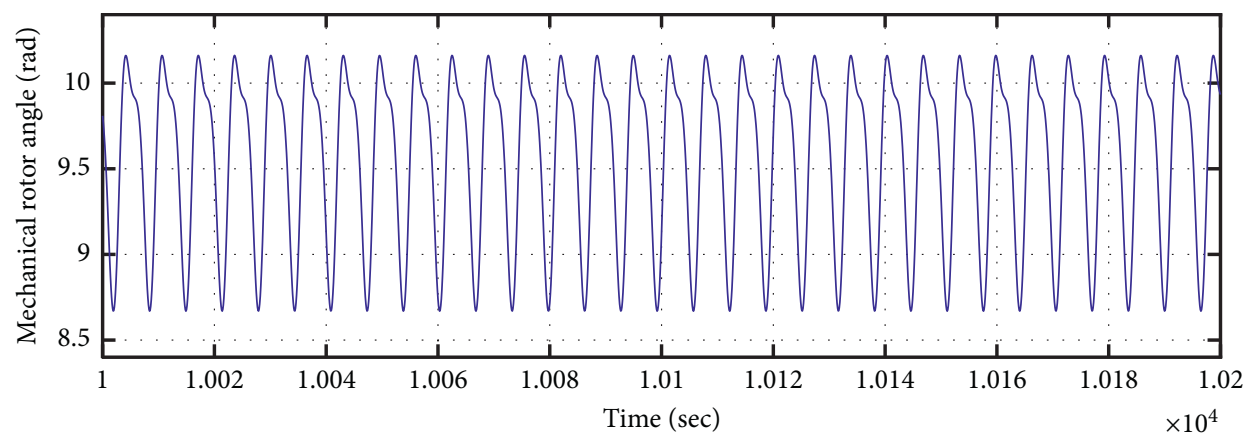

(a)

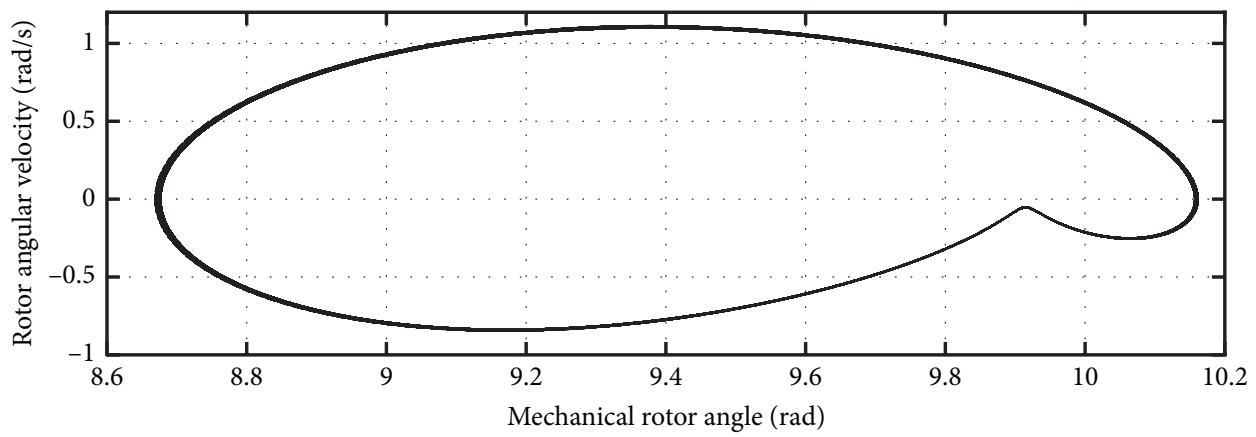

(b)

Figure 11: A square-wave dither signal was injected to control the chaotic motion of the power system for $W=0.2$ and $K_{f}=0.009$ : (a) time responses of the controlled system; (b) phase portrait of the controlled system. 


\section{Conclusions}

This work addressed the rich nonlinear dynamics and chaos control in power systems. The resulting bifurcation diagram showed many nonlinear behaviors, indicating that the tested power system exhibited chaotic motion at lower $K_{f}$ meaning that the system could undergo a cascade of period-doubling bifurcations prior to the onset of chaos. Numerical approaches, including phase portraits, Poincaré maps, and frequency spectra, were employed to explore the dynamics of the used power system. The most powerful algorithm to determine whether a power system is in chaotic motion or not is to use the Lyapunov exponent and Lyapunov dimension. The presence of a chaotic behavior was generic for certain nonlinearities, parameter ranges, and external forces, and it may need to be avoided or controlled to improve the performance of the power system. The state feedback control scheme was simple and effective for chaos suppression, and it can be implemented by adding the feedback of suitable variables to the original system with sufficient control gain to quench chaos development. Additionally, the square wave of the dither signal can be applied to efficiently convert a chaotic motion into a periodic orbit by injecting a dither signal in front of the nonlinearity of the power system. We believe that studying the nonlinear dynamics and chaos control in power systems will help prevent voltage collapses and advance the development of smart power systems.

Other numerous methods for chaos control have been devised, such as synchronization control, time-delayed feedback control, neurofuzzy control, adaptive control, and bang-bang control. In this study, two chaos control strategies, state feedback control and dither control, were implemented to control the chaotic behavior of a power system. The effectiveness of these proposed chaos control strategies was illustrated through numerical simulations. Also, a simple control method was presented to convert chaos into periodic motion using the linear-state feedback of an available system variable. Overall, it was found that compared with other chaos control methods, the state feedback control technique is simple and can be easily implemented in chaos suppression. Finally, the robustness of the parametric perturbation on a chaotic system with various chaos control methods will be studied in my future work.

\section{Data Availability}

No experimental data are used to support this study.

\section{Conflicts of Interest}

The author declares that there are no conflicts of interest regarding the publication of this paper.

\section{Acknowledgments}

The author would like to thank the Ministry of Science and Technology in Taiwan, Republic of China, for financially supporting this research under Contract No. MOST 1082221-E-212 -010 -MY3.

\section{References}

[1] H. O. Wang, E. H. Abed, and A. M. A. Hamdan, "Bifurcations, chaos, and crises in voltage collapse of a model power system," IEEE Transactions on Circuits and Systems I: Fundamental Theory and Applications, vol. 41, no. 3, pp. 294-302, 1994.

[2] I. G. Adebayo and Y. Sun, "Performance evaluation of voltage stability indices for a static voltage collapse prediction," in Proceedings of the 2020 IEEE PES/IAS PowerAfrica, October 2020.

[3] R. Tiwari, K. R. Niazi, and V. Gupta, "Line collapse proximity index for prediction of voltage collapse in power systems," International Journal of Electrical Power \& Energy Systems, vol. 41, no. 1, pp. 105-111, 2012.

[4] R. M. M. Pereira, C. M. M. Ferreira, and F. P. M. Barbosa, "Comparative study of STATCOM and SVC performance on dynamic voltage collapse of an electric power system with wind generation," IEEE Latin America Transactions, vol. 12, no. 2, pp. 138-145, 2014.

[5] U. G. Vaidya, R. N. Banavar, and N. M. Singh, "A chaotic phenomenon in the damped power swing equation," in Proceedings of the 38th IEEE Conference on Decision and Control, pp. 4650-4655, Phoenix, AZ, USA, August 1999.

[6] Y. Yu, H. Jia, P. Li, and J. Su, "Power system instability and chaos," Electric Power Systems Research, vol. 65, no. 3, pp. 187-195, 2003.

[7] L. Kuru, E. Kuru, and M. A. Yalcin, "An application of chaos and bifurcation in nonlinear dynamical power systems," in Proceedings of the 2nd International IEEE Conference on Intelligent Systems, Varna, Bulgaria, June 2004.

[8] H. Gholizadeh, A. Hassannia, and A. Azarfar, "Chaos detection and control in a typical power system," Chinese Physics $B$, vol. 22, no. 1, 2013.

[9] X. Wang, Y. Chen, G. Han, and C. Song, "Nonlinear dynamic analysis of a single-machine infinite-bus power system," Applied Mathematical Modelling, vol. 39, no. 10-11, pp. 2951-2961, 2015.

[10] P. M. Vahdati and A. Kazemi, "Bifurcations and chaos in nonlinear dynamics of power systems," in Proceedings of the 24th Iranian Conference on Electrical Engineering (ICEE), pp. 1706-1711, Shiraz, Iran, May 2016.

[11] K. Rajagopal, A. Karthikeyan, P. Duraisamy, R. Weldegiorgis, and G. Tadesse, "Bifurcation, chaos and its control in a fractional order power system model with uncertainties," Asian Journal of Control, vol. 21, no. 1, pp. 184-193, 2019.

[12] H. Ma, F. Min, and Y. Wang, "Nonlinear dynamic analysis and surface sliding mode controller based on low pass filter for chaotic oscillation in power system with power disturbance," Chinese Journal of Physics, vol. 56, no. 5, pp. 2488-2499, 2018.

[13] L. Zhou and F. Chen, "Chaotic dynamics for a class of singlemachine-infinite bus power system," Journal of Vibration and Control, vol. 24, no. 3, pp. 582-587, 2018.

[14] X. Wang, Z. Lu, and C. Song, "Chaotic threshold for a class of power system model," Shock and Vibration, vol. 2019, Article ID 3479239, 7 pages, 2019.

[15] A. Wolf, J. B. Swift, H. L. Swinney, and J. A. Vastano, "Determining Lyapunov exponents from a time series," Physica D: Nonlinear Phenomena, vol. 16, no. 3, pp. 285-317, 1985.

[16] G. Benettin, L. Galgani, A. Giorgilli, and J.-M. Strelcyn, "Lyapunov Characteristic Exponents for smooth dynamical systems and for Hamiltonian systems; a method for computing all of them. Part 1: Theory," Meccanica, vol. 15, no. 1, pp. 9-20, 1980. 
[17] G. Benettin, L. Galgani, A. Giorgilli, and J.-M. Strelcyn, "Lyapunov Characteristic Exponents for smooth dynamical systems and for Hamiltonian systems; A method for computing all of them. Part 2: numerical application," Meccanica, vol. 15 , no. 1 , pp. 21-30, 1980.

[18] E. Ott, C. Grebogi, and J. A. Yorke, "Controlling chaos," Physical Review Letters, vol. 64, no. 11, pp. 1196-1199, 1990.

[19] W. L. Ditto, S. N. Rauseo, and M. L. Spano, "Experimental control of chaos," Physical Review Letters, vol. 65, no. 26, pp. 3211-3214, 1990.

[20] E. R. Hunt, "Stabilizing high-period orbits in a chaotic system: the diode resonator," Physical Review Letters, vol. 67, no. 15, pp. 1953-1955, 1991.

[21] C. Cai, Z. Xu, W. Xu, and B. Feng, "Notch filter feedback control in a class of chaotic systems," Automatica, vol. 38, no. 4, pp. 695-701, 2002.

[22] S. C. Chang and Y. F. Lue, "A study of the nonlinear response and chaos suppression in a magnetically levitated system," Australian Journal of Mechanical Engineering, vol. 18, no. 1, pp. 94-105, 2020.

[23] D. D. A. Costa and M. A. Savi, "Chaos control of an SMApendulum system using thermal actuation with extended time-delayed feedback approach," Nonlinear Dynamics, vol. 93, no. 2, pp. 571-583, 2018.

[24] P. Tooranjipour and R. Vatankhah, "Adaptive critic-based quaternion neuro-fuzzy controller design with application to chaos control," Applied Soft Computing, vol. 70, pp. 622-632, 2018.

[25] O. I. Tacha, C. K. Volos, I. M. Kyprianidis, I. N. Stouboulos, S. Vaidyanathan, and V.-T. Pham, "Analysis, adaptive control and circuit simulation of a novel nonlinear finance system," Applied Mathematics and Computation, vol. 276, no. 5, pp. 200-217, 2016.

[26] O. Tutsoy and M. Brown, "Chaotic dynamics and convergence analysis of temporal difference algorithms with bang-bang control," Optimal Control Applications and Methods, vol. 37, no. 1, pp. 108-126, 2016.

[27] M. J. Liu and Z. L. Piao, "Study on chaos control for nonlinear power system," in Proceedings of the IEEE International Workshop on Intelligent Systems and Applications, Wuhan, China, May 2009.

[28] M. Kumar and P. P. Singh, "Chaos control of an extended four dimensional fundamental power system using sliding mode control," in Proceedings of the 5th IEEE Uttar Pradesh Section International Conference on Electrical, Electronics and Computer Engineering (UPCON), November 2018.

[29] C. Wang, H. L. Zhang, W. H. Fan, and P. Ma, "Finite-time function projective synchronization control method for chaotic wind power systems," Chaos, Solitons and Fractals, vol. 135, pp. 1-12, 2020.

[30] J. Wang, L. Liu, C. Liu, and X. Li, "Adaptive sliding mode control based on equivalence principle and its application to chaos control in a seven-dimensional power system," Mathematical Problems in Engineering, vol. 2020, Article ID 1565460, 13 pages, 2020.

[31] C. Cai, Z. Xu, and W. Xu, "Converting chaos into periodic motion by state feedback control," Automatica, vol. 38, no. 11, pp. 1927-1933, 2002.

[32] C. C. Fun and P. C. Tung, "Experimental and analytical study of dither signals in a class of chaotic system," Physics Letters A, vol. 229, no. 4, pp. 228-234, 1997.

[33] N. Kopell and R. Washburn, "Chaotic motions in the twodegree-of-freedom swing equations," IEEE Transactions on Circuits and Systems, vol. 29, no. 11, pp. 738-746, 1982.
[34] S. C. Chang, "Study of Structural Stability for Power System," M.S. thesis, National Central University, Taoyuan City, Taiwan, 1992.

[35] IMSL, Inc User's Manual-IMSL MATH/LIBRARY, 1989.

[36] J. L. Kaplan and L. A. York, "Chaotic behavior of multidimensional difference equations," Lecture Notes in Mathematics, Springer, New York, NY, USA, 1979.

[37] Y. M. Liaw and P. C. Tung, "Application of the differential geometric method to control a noisy chaotic system via dither smoothing," Physics Letters A, vol. 239, no. 1-2, pp. 51-58, 1998.

[38] P. A. Cook, Nonlinear Dynamical Systems, Prentice-Hall, Upper Saddle River, NY, USA, 1994. 